\title{
Enhanced assessment of limb neuro-mechanics via a haptic display
}

\author{
Alejandro Melendez-Calderon ${ }^{1,6^{*}+}$, Davide Piovesan ${ }^{2,6+}$, James L Patton ${ }^{1,3,4,5,6}$ and Ferdinando A Mussa-Ivaldi ${ }^{1,4,6,7}$
}

\begin{abstract}
Current state-of-the-art quantitative assessments of abnormal neuro-mechanics (e.g., spasticity, rigidity, dystonia) require sophisticated measurement systems that, together with the lengthiness of the data acquisition, make these approaches impractical for the clinical setting. Our long-term goal is to create a tool that enhances the capability of therapists to diagnose abnormal neuro-mechanics without the need of lengthy procedures and sophisticated equipment. Towards this direction, we present a haptic display of abnormal limb neuro-mechanics. This haptic display can be used as a training device for clinicians to understand the causality of the haptic sensation associated with neurological impairments and abnormal biomechanics during manipulation of a patients' limb. Other uses include the testing of operational research algorithms to maximize the exchange of haptic information, reproducing conditions of human-human interaction, and a test bed for developing novel assessment techniques and targeted interventions. Experimental results support the hypothesis that the best assessment of abnormalities can be obtained via specific manipulation strategies.
\end{abstract}

Keywords: Biomechanics; Haptic display; Hypertonia

\section{Background}

A common consequence of many neurological disorders like cerebral palsy, Parkinson's disease, or stroke (to name a few) is the appearance of atypical neuro-mechanical conditions such as an increase in muscle tone (hypertonia) due to active contraction of the muscle, hyperexcitability of motoneurons causing excessive co-activation, muscle contractures, etc. It is common in today's clinical practice to manipulate the patient's limbs to diagnose these abnormal neuro-mechanical changes. Through such physical interaction, clinicians extract as much information as possible to infer the patients' condition, such as in the assessment of hypertonic conditions [1-3] (e.g., spasticity, rigidity, dystonia, among others). This approach is subjective [4], which may be a limitation when inferring the nature and gravity of the impairment since the effect generated by pluri-articular muscles to multi-joint mechanics can be misjudged. A misjudgment on the

\footnotetext{
* Correspondence: alejandro.melendez@northwestern.edu

${ }^{\dagger}$ Equal contributors

${ }^{1}$ Department of Physical Medicine and Rehabilitation, Northwestern

University, 60611 Chicago, IL, USA

${ }^{6}$ Sensory Motor Performance Program, Rehabilitation Institute of Chicago, 60611 Chicago, IL, USA

Full list of author information is available at the end of the article
}

initial or intermediate assessments can lead to selecting a non-optimal intervention, which can have negative consequences, such as increasing intervention time, rising in treatment cost, or even worsening the condition. Hence, the capability to discriminate between different types and levels of impairments is important for the clinical intervention to be chosen and planned accordingly.

Traditional engineering approaches to assess differences in limb mechanics such as non-parametric $[5,6]$ and parametric $[7,8]$ system identification techniques provide a less subjective, quantitative measure of biomechanical variables. However, the necessity of sophisticated measurement systems, such as stiff robotic devices, makes most of these classical engineering methods impractical for the clinical setting due to a high cost/benefit ratio. In addition, the majority of studies applying such purely roboticdriven approaches have been limited to single joints (e.g., knee, ankle, elbow) as more complex joints (e.g., shoulder) pose significant challenges [9]. Yet, abnormal neuro-mechanical conditions such as hypertonia can encompass alteration of inter-muscular (heteronymous) reflexes [10] or abnormal multi-joint couplings due to increased rigidity [11,12]. Consequently, some of the

\section{Springer}


most important clinical problems have been ignored in these engineering approaches.

\section{Our approach}

A recent review on spasticity assessment [13] concluded that there is a need for simple instruments that can provide reliable measures in a practical setting. Therefore, our goal is to extend the interactive nature of traditional assessment techniques by providing clinicians with a method that enables them to appreciate and quantify hypertonic phenomena during manipulation of the affected limb in multiple degrees of freedom (DOF). Towards this end, we propose a haptic display, in which a model of an impaired limb is haptically rendered on a robotic device in which clinicians can physically interact with. This haptic display allows us to evaluate the threshold of human perceptions to different pathological neuro-mechanics at different levels of severity, which in turn can translate into different strategies that enhance the human perception of these impairments. This will likely provide therapists with better training and improved classification, measurement, and treatment of these disorders.

Haptic rendering of physical impairments of stroke survivors have been reported previously (e.g., [14,15]). Such approaches, however, have been limited to only one DOF and have focus on mimicking current clinical assessment techniques (e.g., Ashworth test). It is essential to extend the manual assessment of hypertonia to more than one DOF so as to capture the effect of pluriarticular muscles and multi-joint coupling. Differently from the previous work, our approach allows us to characterize how clinicians perceive different types of hypertonia in a multi-DOF environment. The interaction in our haptic display occurs through a single haptic port under the rationale that assessment techniques derived from the use of this tool should be directly applicable to both on-site and remote scenarios (Figure 1). Extending traditional manipulation methods in tele-interaction scenarios requires the use of an exoskeleton on the patient side and a robotic mock-up of the patient's limb on the clinician side so that the different limb segments can be manipulated independently. We suggest the implementation of simpler configurations where the interaction occurs through a single port from both sides, allowing the use of commercially available robots. Our hypothesis is that the individualization of abnormalities through a single interaction port in patientclinician physical interaction can be done more objectively via specific manipulation strategies (probing motions) derived from understanding the forces at the point of interaction produced by the different types of impairment. The force-motion generated by the patient-clinician interaction along these motions could uniquely characterize the nature and severity of the impairment. We expect that our haptic display will help us better understand how to improve human perception during physical manipulation.

\section{Methods}

\section{Haptic display of limb neuro-mechanics}

The haptic display comprises a multi-joint robotic manipulator that is programmed to output mechanical forces that match the forces a clinician would feel when moving a limb (e.g., arm, leg, hand) of a patient (model). To interact with the model, subjects seat in front of the robot and hold the robot's end effector. The end effector of the robot is virtually connected to the model via a two-way connection that can be rendered, for instance, through a shared virtual environment which includes a simulated mechanical element $[16,17]$. The limb model

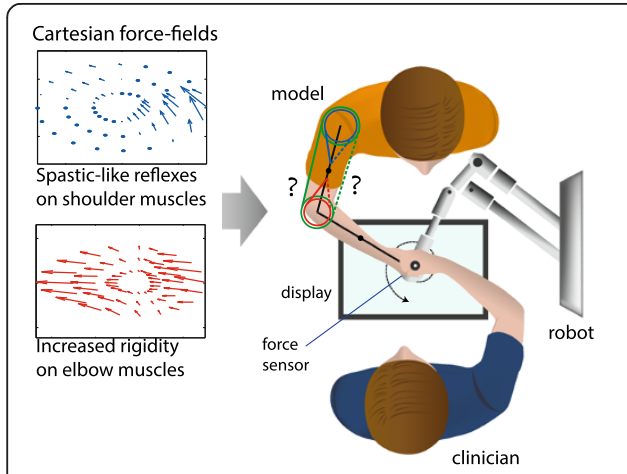

a) Training

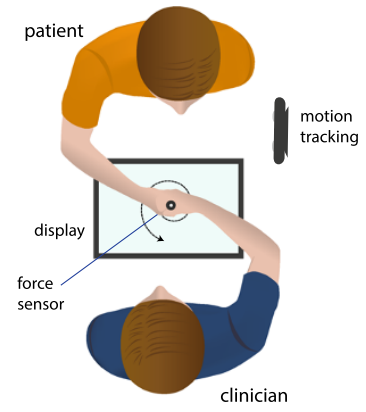

b) On-site assessment

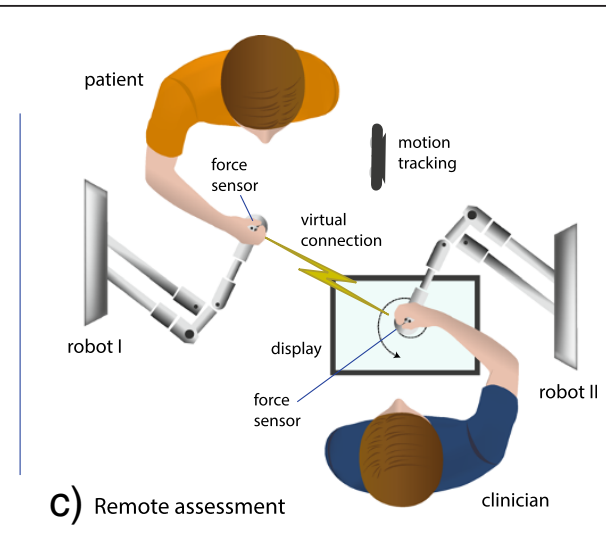

C) Remote assessment

Figure 1 Different uses of the proposed method include assessment training and on-site and remote assessment scenarios. (a) Training - a clinician interacting with a simulated patient rendered on a robotic device (haptic display). Different abnormal biomechanics can be rendered by the haptic display so the therapist can be trained in assessing these conditions. Manipulation techniques for assessment derived from the use of the haptic display can be extended to both on-site and remote scenarios. (b) On-site assessment - a clinician interacting with a patient using a system to assist in the assessment and determination of the abnormal limb biomechanics of a patient. (c) Remote assessment - a clinician interacting with a patient using a non-co-located setup of the system described in (b). 
is rendered as if the patient were seated opposite the participants or at any other desired configuration. Both the subjects' hand and the robotic arm can be covered by an opaque horizontal screen, on which an image of the rendered virtual limb is projected. A superimposed image representing the workspace can also be projected. This configuration can also be reproduced during teleassessment, where instead of a simulated arm, the users interact remotely with another person.

\section{Neuro-mechanical model of a human limb}

The force produced by muscles can be expressed in terms of passive (i.e., intrinsic physical properties) and active (i.e., reflexes and voluntary activation) components. The combination of forces generated by different muscle groups gives rise to characteristic force patterns at a limb's endpoint. The set of forces associated with a set of limb states (e.g., position, velocity) in the Cartesian space is a force field $[18,19]$. In our haptic display, the force of each muscle group is modeled as a linear combination of non-linear passive and active components. The passive component is position dependent and it is a function of the muscle length, whereas the active component depends on reflexive activation and is both position and velocity dependent. The resulting force field is the linear superposition of the two force components. This simple, yet representative, approach allows us to simulate different normal and hypertonic-like forces at the point of interaction by modifying few weighting coefficients. The different abnormal force fields generated by a hypertonic limb can be rendered on a robotic device (haptic display) and so the haptic perception to the nature and severity of hypertonic phenomena can be investigated systematically (e.g., [20,21]).

As a proof of concept, we emulated a simple representation of the human arm with six different muscle groups that are embedded in a planar, two-joint mechanism. The model considers rigid body dynamics and muscle intrinsic and active properties. The model was implemented in Simulink and was rendered on a two revolute joint planar robot running in real-time using $\mathrm{xPC}$ Target at a rate of $1 \mathrm{kHz}$. The dynamics of the virtual arm moving in a horizontal plane while interacting with the subject were modeled as

$$
\begin{aligned}
H[q] \ddot{q}+C[q, \dot{q}] \dot{q}= & J_{q}{ }^{T}[q] \cdot F_{\text {external }} \\
& -J_{\lambda}{ }^{T}[q] \cdot(\Phi[\lambda, u[\dot{\lambda}]]+\Psi[\lambda])
\end{aligned}
$$

where $H[q]$ represents the arm inertia matrix of a double pendulum system; $q$ denotes the vector of shoulder and elbow joint angles [rad]; $C[q, \dot{q}] \dot{q}$ is the term corresponding to Coriolis and centripetal forces; $J_{q}[q]$ is the
Jacobian matrix transforming an endpoint force, $F_{\text {external, }}$ into joint torque; $\lambda$ and $\lambda$ correspond to the muscle stretch and muscle stretch velocity, respectively; $\Phi$ is a vector of muscle forces produced by motor signals $u$; and $\psi$ is a vector of muscle forces that are intrinsic to the biomechanical properties of the muscle. Simulationspecific parameters for the limb centers of mass and inertia were estimated using anthropometrical tables from Winter [22]. The Jacobian matrix $J_{\lambda}[q]$ transforms the muscle force into joint torque and contains the muscle moment arms $\rho$ at any particular position, $q$ :

$$
J_{\lambda}[q]=\left(\begin{array}{cccccc}
-\rho_{\mathrm{sf}} & \rho_{\mathrm{se}} & 0 & 0 & -\rho_{\mathrm{bf}_{1}} & \rho_{\mathrm{be}_{1}} \\
0 & 0 & -\rho_{\mathrm{ef}} & \rho_{\mathrm{ee}} & -\rho_{\mathrm{bf}_{2}} & \rho_{\mathrm{be}_{2}}
\end{array}\right)^{T}
$$

The sub-indexes correspond to sf, shoulder adductors (deltoid anterior, coracobrachialis, pectoralis major clav.); se, shoulder abductors (deltoid posterior); ef, elbow flexors (biceps long, brachialis, brachioradialis); ee, elbow extensors (triceps lateral, anconeus); bf, bi-articular flexors (biceps short); and be, bi-articular extensors (triceps long) muscle groups.

The force of each muscle group is modeled as a linear combination of active (i.e., produced by a motor command $u$ ) and passive (i.e., produced by intrinsic rigidity of the muscles and connective tissue) components [23,24] associated with intrinsic rigidity of the muscles and connective tissue. The force produced by each muscle in response to the motor signal $u$ is

$$
\begin{aligned}
& \Phi[\lambda, u[\dot{\lambda}]]=\left(\begin{array}{llllll}
\varphi_{\mathrm{sf}} & \varphi_{\mathrm{se}} & \varphi_{\mathrm{ef}} & \varphi_{\mathrm{ee}} & \varphi_{\mathrm{bf}} & \varphi_{\mathrm{be}}
\end{array}\right)^{T} \\
& \varphi_{i}=\max \left(\begin{array}{l}
0, \\
\alpha_{i} u\left[\dot{\lambda}_{i}\right] \cdot \Gamma \cdot e^{-\Gamma^{2}}
\end{array}\right) \\
& \Gamma=\left(\frac{\lambda_{i}-\lambda_{\min , i}}{\lambda_{\max , i}-\lambda_{\min , i}}-\frac{\lambda_{\text {rest }, i}}{\lambda_{\max , i}}\left(1-u\left[\dot{\lambda}_{i}\right]\right)\right)
\end{aligned}
$$

The term $u$ corresponds to an active motor signal (e.g., reflexes, voluntary commands); the term $\alpha$ corresponds to the stiffness of the muscle when the system is linearized at its equilibrium position when the activation is maximum (i.e., $u=1$ ).

Typical assessment of hypertonia is performed by asking the patient to not voluntarily intervene. Under this assumption, the term $u[\dot{\lambda}]$ corresponds to an active motor signal that depends on the muscle stretch velocity $\dot{\lambda}$ (e.g., tonic reflex), which we defined as

$$
u\left[\dot{\lambda}_{i}\right]=\beta_{i} \min \left(1, \max \left(0, \frac{\dot{\lambda}_{i}}{\dot{\lambda}_{\max , i}}\right)\right) \mid \beta_{i} \in[0,1]
$$

where $\beta$ corresponds to a 'stretch gain'. The variables 
$\lambda_{\text {rest }, i}, \lambda_{\max , i}$, and $\lambda_{\max , i}$ correspond to the length of the $i$ thmuscle group at rest, its maximum length and maximum rate of length change, respectively. The maximum rate of length change was calculated as the one obtained by moving the endpoint of the virtual arm along a circle of $35 \mathrm{~cm}$ in diameter at a frequency of $2 \mathrm{~Hz}$. The maneuver was assumed to be a good compromise between the amplitude and frequency of a very fast movement in endpoint space.

The force produced by the intrinsic rigidity of the muscles and connective tissue is a function of the muscles' lengths:

$$
\begin{aligned}
& \Psi[\lambda]=\left(\begin{array}{llllll}
\psi_{\text {sf }} & \psi_{\text {se }} & \psi_{\text {ef }} & \psi_{\text {ee }} & \psi_{\text {bf }} & \psi_{\text {be }}
\end{array}\right)^{T} \\
& \psi_{i}=\max \left(\begin{array}{l}
0, \\
K_{m, i}\left(\lambda_{i}-\lambda_{\text {rest }, i}\right) e^{-\left(\lambda_{i}-\lambda_{\text {rest }, i}\right)^{2}}
\end{array}\right)
\end{aligned}
$$

The term $\Psi[\lambda]$ can be multiplied by a generalized logistic function to avoid sharp discontinuities when adding both passive and active components in Equation 1. The term $\lambda$ represents the muscle length, and $K_{m}$ corresponds to the muscle stiffness linearized at the muscle's rest position.

\section{Haptic rendering of abnormal limb neuro-mechanics}

Haptic rendering of the correct ranges of forces that impaired individuals can exhibit is essential so that valid psychometric studies on haptic perception can be done in a haptic display. These ranges of forces depend on both the intrinsic physical properties (rigidity) of the limb and the influence of muscle reflexes on the limb mechanics. However, the separation of these two terms is an open, difficult, and controversial issue [6,25-28]. However, assuming that both intrinsic and reflexive stiffness are linearly combined, we could get a good approximation of the order of magnitude of these two terms by means of measuring postural and stiffness maps. A postural map represents the stationary force field (i.e., with zero velocity) produced by the intrinsic properties of the limb measured at specific locations $[11,29,30]$. It is representative of the intrinsic properties of the arm. The integral of this force field with respect to the position workspace is the potential energy associated with the passive mechanics of the limb. A stiffness map represents the Hessian of the potential of active muscle force at a specified velocity obtained by perturbing the limb at different positions and frequencies. Given the rapid change in velocity during the acquisition of these maps, the reflexive stiffness is expected to rise and be somehow distinguishable from the intrinsic rigidity, as demonstrated in [11]. By integrating each map twice with respect to the position, a potential describing the active component of the muscles at the specified velocity is obtained.

Given a database of different force fields generated by different impaired individuals in the form of postural and stiffness maps, one can use optimization algorithms (e.g., simulated annealing) to tune the sensitive parameters of the model, such as the stiffness of the connective tissue and the reflex gain for each muscle, to generate a force field that matches the measured ones (see Figure 2). Therefore, one could create and reproduce any arbitrary mixture of representative reflex and intrinsic stiffness by means of our haptic display.

\section{Haptic rendering of hypertonic-like forces}

Among the numerous factors that characterize hypertonia, we are interested in training the ability to discriminate forces produced by increased muscle rigidity and the nonlinear phenomena associated with velocity-dependent stiffness. Another way of simulating hypertonic-like forces is to assume that these could be achieved by increasing both $\alpha$ and $K_{m}$. in Equations 3 and 5. Measurements of joint rigidity during passive movements are available in the literature for both stroke survivors and unimpaired individuals $[8,31]$. Based on such data, the average joint passive linear stiffness of unimpaired individuals can be taken as the lower boundary of joint rigidity. The upper boundary of joint rigidity corresponds to the passive linear stiffness recorded on stroke survivors with the Modified Ashworth Score equal to $4[11,32]$. To render intermediate levels of severity, one could consider the just noticeable difference (JND) or the Weber fraction, which is an important index representing the sensitivity of the subject to stiffness stimuli. Different stiffness JND have been obtained empirically, depending on the experimental protocol used. For palpation with a fixed displacement, the value of stiffness JND is around 8\% [33]. For free exploration, the JND is much higher and can be up to $67 \%$ [34]. Since the rigidity discrimination in the clinical setting is performed with a free movement, a stiffness JND $=60 \%$ could be chosen to segment the whole range of rigidity between the two aforementioned boundary conditions.

\section{Psychophysical experiments}

We used our haptic display to investigate whether specific exploration patterns or probing motions can be used to discriminate the salient features of a given set of force fields associated with different hypertonic-like conditions. In particular, we present a study that evaluates the capabilities of (non-therapists) individuals in discriminating between different hypertonic virtual arms with diverse muscle groups affected. Three different muscle groups were tested - $i$ ) hypertonic shoulder muscles, ii) hypertonic elbow muscles, and iii) hypertonic biarticular muscles - at four levels of severity - i) very mild, ii) mild, iii) moderate, and iv) severe, following a Weber Fraction of 0.6.

Twelve subjects (non-therapists), naïve to the experimental conditions, participated in the study. Subjects 


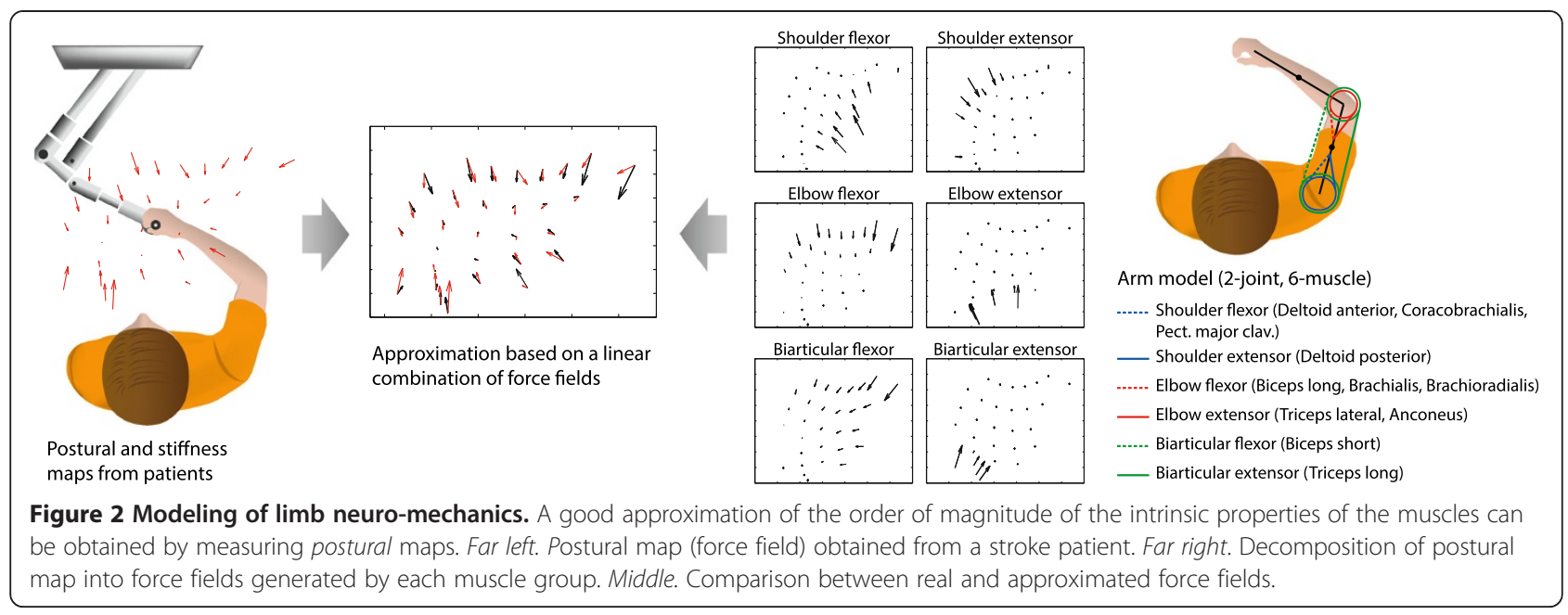

gave informed consent prior to participation. Experiments were approved by the Northwestern University's Institutional Review Board. Participants were divided in two groups: i) non-instructed and ii) instructed. Noninstructed subjects were required to discriminate between the different conditions using their own-developed manipulation technique during a familiarization phase. Instructed subjects were required to manipulate the haptic display with specific probing motions that highlight the salient features of each abnormal condition (pulling and semielliptical motions - see 'Selection of specific probing motions').

\section{Experimental protocol}

The experiment was divided in two consecutive phases: a) familiarization and b) assessment. During familiarization, participants interacted with four different virtual arms: i) unimpaired arm, ii) maximally impaired shoulder, iii) maximally impaired elbow, and iv) maximally impaired bi-articular muscles. Subjects interacted sequentially with each condition in blocks of $30 \mathrm{~s}$ for eight trials ( 8 trials $\times 4$ conditions $=32$ trials). During this phase, a legend appeared on the top right corner of the screen indicating the condition that the user was experiencing. In order to familiarize with the different haptic sensations produced by the hypertonic muscle groups, non-instructed subjects were free to interact with those four conditions as they wished, while instructed subjects were specifically asked to move the arm with a pulling and semi-elliptical probing motions. During assessment, all subjects were randomly presented with different abnormal virtual arms of varied hypertonic severity. Their task was to identify which group of muscles was impaired. All subjects were presented each condition nine times in trials of $15 \mathrm{~s}$ (3 hypertonic conditions $\times 4$ levels of severity $\times 9$ trials $=108$ trials). The only information available during this phase was the configuration of the arm and the rendered force at the end effector of the haptic display. After physical manipulation of the virtual arm, subjects were required to select one of the three options presented on the screen - shoulder, elbow, or both. Participants could take as much time as they wanted to give their assessments and start a new trial. The whole experiment lasted for about $45 \mathrm{~min}$. Simulation-specific parameters were the same as the ones described in detail in [20].

\section{Selection of specific probing motions}

As a first approximation, we planned the probing motion for this experiment considering only the effect of the position-dependent muscle force $\Psi$. Given the three force fields associated with severe hypertonic shoulder, elbow, and bi-articular muscle groups, our first goal was to find the minimum number of probing motions that, executed within each force field, would produce a different force profile. Such force profiles along the trajectories ought to be easily identifiable from one another. One possible strategy is to include the locations of the workspace in which the minimum and maximum force magnitude occurs for each field in each probing trajectory. We called this strategy the min-max paradigm. The set of probing trajectories should be designed so that, along the same probing motion, the minimum and maximum force magnitude occurs at different locations for each force field. Figure 3(a) represents the passive force $\Psi$ for severe hypertonic muscle groups, generated by a circular probing motion. It can be seen that the minimum and maximum force occurs in different locations along the circles for hypertonic shoulder and elbow force fields. Yet, there is not much difference in the location between hypertonic shoulder and bi-articular. Hence, an extra probing motion would allow disambiguation between conditions. It can be demonstrated that given $n$ types of force fields, when using the min-max paradigm, it is 


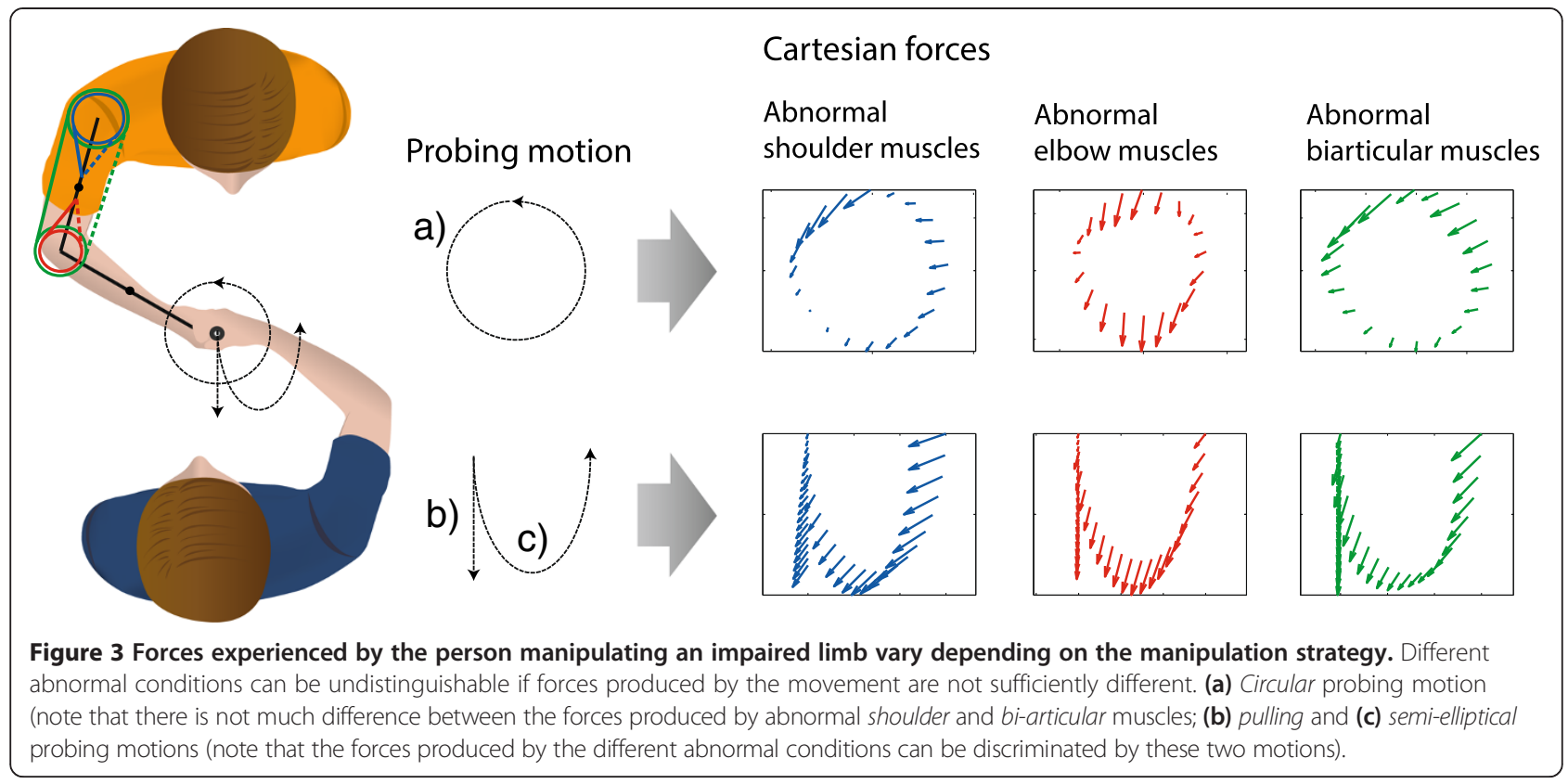

always possible to identify all impairments by exploring $n-1$ trajectories.

While circles could completely characterize the location and intensity of the maximum force associated with a specific impairment, the path to be covered is quite long. This is a limitation since the effect of the reflexes is attenuated after prolonged repetitive movements. To avoid this, we tried to reduce the length of the movements by incorporating simple strokes. According to the min-max paradigm, the arm's equilibrium position (i.e., the center of each circle) ought to be included in the probing motion as it is the point of absolute minimum force for all conditions. On the other hand, it should be noted that the magnitude of the force is not the only variable that can be discriminated. The orientation of the force is also important as it allows for an unequivocal haptic cue. Pulling the endpoint of impaired arm from its equilibrium position along the mid-sagittal plane in a fast and straight motion (Figure 3(b)), we can find that the orientation of the force profile generated by the hypertonic shoulder force field is completely different from those produced in the other two fields. Hence, we chose this movement for discriminating the shoulder force field from all the other. It is important to notice that the orientation and magnitude of the force would change depending on the impairment. We refer to these probing strategies as the orientation paradigm. By pulling the endpoint of impaired arm along a semi-elliptical trajectory as shown in Figure 3(c), the orientation of the corresponding force profile is quite different if the movement is executed when interacting with hypertonic elbow or bi-articular conditions. This is the second trajectory that we chose to complete the set of probing motion. Since the number of impairments was restricted to three in the current experiment, we hypothesize that subjects would be able to discriminate between the conditions by using only two probing motions.

\section{Results and discussion \\ Psychophysical experiments}

Figure 4a shows stereotypical probing motions executed by both groups, and Figure $4 \mathrm{~b}$ shows a comparison of the reliability between the two manipulation techniques. It can be seen that the reliability of the users' discrimination was better when they followed a specific probing motion as compared to random exploration. These experimental results support the hypothesis that better assessment of abnormalities can be obtained via specific probing motions that highlight haptic features of the different abnormalities, thus enhancing human perception.

\section{Other experiments}

In addition to this experiment, we are currently using this haptic display to $i$ ) assess the capabilities of naive subjects and expert therapists to correctly identify the nature and severity (see $[20,21]$ ) of hypertonic impairments and to $i$ i) assess how the perception of hypertonia is affected by the impedance introduced by the virtual connection between a patient and a clinician interacting remotely [35]. The haptic display allows to tackle several practical issues. For example, it can be used to investigate the effects of transmission delays on haptic perception of abnormal biomechanics or to derive specific probing motions that would enhance recognition of such disorders. 


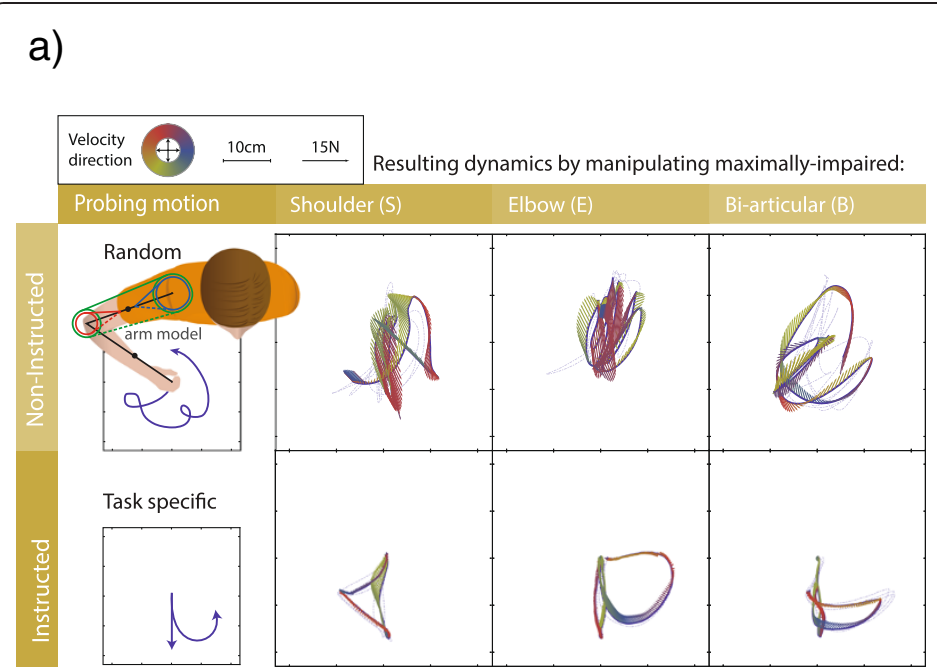

b)

Figure 4 Better assessment of abnormal neuro-mechanics can be obtained via designed probing motions. (a) Stereotypical probing motions executed by experimental subjects. Non-instructed subjects manipulated the hypertonic arm model mainly with random motions. Instructed subjects were encouraged to use the two task-specific probing motions (pulling and semi-elliptical motions - see 'Selection of specific probing motions'). (b) Mean and standard error ( $n=6$ per group) of the assessment reliability as calculated in [20]; specific probing motions significantly improved the assessment. Note that a random guess approach has a reliability of 1/3.

\section{Discussion}

This paper presents a haptic display for assessing neuromechanical pathologies such as hypertonia. An innovative step in our haptic display is to give clinicians the capacity to translate clinical parameters into a haptic sensation. This could also fulfill the need for a training system that can help clinicians to better understand the causality of the haptic sensation experienced during physical manipulation of a patient's limb afflicted by a particular impairment, for instance, to find specific probing motion(s) that are better at discriminating between different pathologies. While in current clinical practice the assessment of different aspects of hypertonia are expressed by the subjective judgment of the therapist [4], to our knowledge, there is no scientific literature that has systematically investigated a therapist's perceptual identification of the nature and severity of multi-joint hypertonic phenomena. Some studies have tried to categorize the sensitivity of therapists to nonbiological stiffness [36,37]; however, these studies have been based on identifying the sensitivity of tactile sensations produced by surfaces. Our haptic display allows us to systematically study these conditions.

\section{A guided approach to manipulation}

The capability to discriminate between two haptic stimuli is not just a function of touch sensitivity. While direct touch conveys cutaneous information about local surface features at the points of contact, probing movements (such as in hypertonia assessments) convey information mostly from proprioceptors [38]. The manner in which a user chooses to actively probe can influence the information gained and quality of the assessment. Visually impaired individuals use different exploration strategies which allow them to gain richer information content, and when sighted subjects are required to use similar strategies, their performance is radically improved [39]. Our haptic display is therefore a valuable tool to explore the haptic sensation of different neuro-mechanics and to determine the most informative probing motion(s) to be used in order to discriminate them

It should be noted, however, that the number of probing motions to distinguish different abnormalities could be quite large and the search could be long and tedious. This poses a problem, as humans can handle only limited amounts of information at once and their memory is often not reliable. Our current research focuses on the development of an operational research algorithm that searches for the 'most informative probing motion', i.e., the one that produces the most characteristic haptic sensation when interacting with different stereotypical force fields generated by patients.

Autonomous guided vehicles operating in dynamic and unknown environments are often faced with the problem of deciding where to go to get the most relevant information [40]. The dynamic environment is assumed to be unknown, but the automaton has a sensor to measure the environment. The environment can be described using a potential function such as an elastic energy, where maxima of the potential are associated with repulsive forces which represent obstacles to avoid. Minima in the potential correspond to attractive forces representing waypoints.

This framework closely resembles the problem a clinician faces when testing a patient. The total potential, which gradient is composed of the characteristic forces 
of the patient's hypertonic arm, is unknown to the clinician (i.e. they need to be assessed). Conversely, the clinician has the capability to 'perceive' the force generated by hypertonic arms, as the autonomous guided vehicles can sense the environment. We wish for the clinician to learn the structure of the patients' total potential by identifying the areas within the subjects' reachable space that are dynamic and the rate of change for these areas. Given the structure of the total potential, we conceive a path for the clinicians to allow them sensing such dynamic areas.

\section{Future directions}

\section{Non-robotic assessment techniques}

A derivation from the knowledge gained from the studies using our haptic display is an assessment approach in which clinicians and computational algorithms participate in the diagnosis process. The idea is to have clinicians physically interacting with the patient while kinematic and dynamic information is fed into a computational algorithm. The algorithm would suggest optimal manipulation strategies to the clinician for the identification of the impairment. These optimal strategies would consider the non-linear effects of human perception and stereotypical forces produced by the different types of impairment.

An expert clinician's experience should provide an educated guess of what the impairment is. This can be immediately translated into a total potential that can be searched by the informative path planning algorithm, which will suggest to the clinician the best trajectory to follow for the assessment.

Our goal is to close the loop between human experts and a database of stereotypical force fields generated by different types of impairment via a mathematical algorithm, essentially augmenting human perception and interpretation capabilities with computer algorithms. The therapist would be able to select the initial choices of most probable impairments, and the algorithm will suggest a minimum amount of probing for identification. This way, the experience of the therapists will be embedded in a supervised algorithm, where both human and classification algorithms interact. This approach would allow us to identify exploration strategies (easily recognizable in intensity, location, and time course) that maximize exchange of information and can uniquely discriminate among different types of hypertonia. In addition, this would provide clinicians with the capability of testing a patient easily in a routine clinical setting with the ability to support the perceptual findings by quantitative methods. This approach is innovative as it shifts the analysis of impaired behavior from the mere perception of force to the characterization of contact forces, motions, and haptic perception produced in the patienttherapist interaction.

\section{Robotic-aided targeted intervention}

Given a set of possible interventions to treat specific impairments, clinicians could analyze their impact directly on the haptic display. For example, two common interventions for the treatment of severe hypertonia in children cerebral palsy are surgery or injections of botulin toxin. The invasiveness of these procedures makes them a one-time intervention. Therefore, careful planning is required. With our haptic display, clinicians could test the biomechanical effects of the different kinds of intervention without even touching the patient. This can be of great help on these cases, helping clinicians to take a more reliable decision and with less margin of error.

\section{Conclusions}

We propose a haptic display that can serve as an aiding tool to $i$ ) synthesize the impairment based on direct measurements of limb mechanics; ii) train clinicians in diagnosing different types of abnormal biomechanics, affecting different muscle groups; iii) analyze the impact of targeted intervention on specific aspects of limb biomechanics; iv) establish and customize a series of most informative probing motions that would maximize the information regarding the impairment so as to assess abnormal biomechanics in a useful, valid, and reliable manner without the need of sophisticated equipment on-site; or $v$ ) allow the assessment of abnormal biomechanics via remote physical interaction. We expect that this will transform the diagnostic practice by the combination of manual assessment techniques with the computational and mechanical simulation of specific abnormal biomechanics.

\section{Abbreviations}

be: bi-articular extensors (triceps long) muscle groups; bf: bi-articular flexors (biceps short); DOF: degrees of freedom; ee: elbow extensors (triceps lateral, anconeus); ef: elbow flexors (biceps long, brachialis, brachioradialis); JND: just noticeable difference; se: shoulder abductors (deltoid posterior); sf: shoulder adductors (deltoid anterior, coracobrachialis, pectoralis major clav).

\section{Competing interests}

Part of the work in this manuscript has been used to redact the following provisional patent application: U.S. Patent App. No. 61/991,370, HAPTIC DEVICE AND METHODS FOR ABNORMAL LIMB BIOMECHANICS (2014) to AM-C, DP and FAM-I. JLP has no competing interests. AM-C is a current employee at Hocoma AG, a medical technology company. However, the work presented in this paper has been performed before any relationship between $\mathrm{AM}-\mathrm{C}$ and Hocoma AG.

\section{Authors' contributions}

AM-C and DP contributed equally to this work. AM-C, DP, and FAM-I conceived the idea. AM-C and DP participated in the rendering of the haptic display. DP and AM-C performed the psychophysical experiments. AM-C and DP analyzed the data. AM-C, DP, JLP, and FAM-I contributed the methods and tools. AM-C and DP wrote the paper. All authors read and approved the final manuscript.

Authors' information

AM-C received a B.Sc. degree in Mechatronics and a Pg.Cert in Control Engineering from Tecnológico de Estudios Superiores de Monterrey-Campus Estado de México (ITESM-CEM) in 2006; an M.Sc. degree in Intelligent Autonomous Systems from Aalborg University in 2007, working on the control of powered transfemoral prostheses; and a PhD degree in Bioengineering 
from Imperial College of Science, Technology and Medicine in 2011, working at the interface of rehabilitation robotics, human-human interaction, and human motor control and learning. He worked as a Postdoctoral Research Fellow at the Rehabilitation Institute of Chicago and Northwestern University from 2012 to 2013. He is currently a Project Manager at Hocoma AG and Adjunct Assistant Professor at the Department of Physical Medicine \& Rehabilitation at Northwestern University. His research has demonstrated how visual cues can compensate for the lack of proprioceptive error and basic mechanisms of human-human physical interaction. His main research interests include human-robot interfaces and motor control/learning.

DP received his M.S.M.E. in 2003 and D.Eng. in Mechanical Measurement in 2007 at the University of Padova, Italy. His dissertation presented a set of experimental and analytical validation techniques for human upper limb models. From 2004 to 2008, he was a visiting scholar and postdoctoral fellow at the Ashton Graybiel Spatial Orientation Lab at Brandeis University, under the supervision of Professors Paul DiZio and James R. Lackner. There, he worked on the mechanics of movement adaptation in non-inertial environments as part of a NASA extramural funding program. He joined Northwestern University in 2008, working as a Postdoctoral Fellow at the Rehabilitation Institute of Chicago under the supervision of Professor Ferdinando (Sandro) Mussa-Ivaldi. DP is currently an Assistant Professor in the Mechanical Engineering Department at Gannon University and a Director of the Biomedical Engineering Program. His main interest is to gain insights on the role of biomechanics in the neural control of movements, with applications to rehabilitation engineering. $J L P$ received a BS degree in Mechanical Engineering and Engineering Science from the University of Michigan, an MS degree in Theoretical Mechanics from Michigan State University, and a PhD degree in Biomedical Engineering from Northwestern University. Currently, he is working as an Associate Professor of Bioengineering at The University of Illinois at Chicago and as an Associate Director of the Center for Rehabilitation Robotics at the Rehabilitation Institute of Chicago (RIC). He also holds affiliate positions in Physical Medicine and Rehabilitation and Mechanical and Biomedical Engineering at Northwestern University. He has worked in manufacturing for Ford and as a cyclotron operator in nuclear medicine before turning his attention to biomechanics and control of human movement. His general interests include robotic teaching, dynamic balance control, haptics, modeling of the human-machine interface, and robot-facilitated recovery from a brain injury. His research has demonstrated how control is made robust by avoiding undesirable situations. His more recent research has demonstrated how one can exploit the nervous system's natural capacity to adapt in order to teach movements and help people to recover from brain injury. He is on the International Advisory Committee for the IEEE-EMBS and also chairs the technical committee on biomedical robotics. FAM-I received a Laurea degree in Physics from the University of Torino, Turin, Italy, in 1978 and a PhD degree in Biomedical Engineering from the Politecnico of Milano, Milan, Italy, in 1987. He is a Professor of Physiology, Physical Medicine and Rehabilitation, and Biomedical Engineering at Northwestern University, IL, USA. He is currently a Senior Research Scientist with the Rehabilitation Institute of Chicago, IL, USA, where he founded and directs the Robotics Laboratory. His areas of interest and expertise include robotics, neurobiology of the sensory-motor system, and computational neuroscience. FAM-I serves on the Editorial Boards of the Journal of Neural Engineering and the Journal of Motor Behavior and is a Member of the Society for Neuroscience and of the Society for the Neural Control of Movement. His achievements include the first measurement of human arm multi-joint impedance; the development of a technique for investigating the mechanisms of motor learning through the application of deterministic force fields; the discovery of a family of integrable generalized inverses for redundant kinematic chains; the discovery of functional modules within the spinal cord that generate a discrete family of force fields; the development of a theoretical framework for the representation, generation, and learning of limb movements; and the development of the first neurorobotic system in which the brainstem of a lamprey controls the behavior of a mobile robot through a closed-loop interaction. He has 120 full-length publications and 100 abstracts.

\section{Acknowledgements}

AM-C and DP were with the Sensory Motor Performance Program, Rehabilitation Institute of Chicago at the time this research was performed. This work was partially supported by NINDS grants 2R01NS035673-10A2 and R01-NS053606-04 and the Coolidge fellowship. The authors thank Felix Huang for the fruitful discussions.

\section{Author details}

'Department of Physical Medicine and Rehabilitation, Northwestern University, 60611 Chicago, IL, USA. 'Biomedical Program, Department of Mechanical Engineering, Gannon University, 16541 Erie, PA, USA.

${ }^{3}$ Department of Bioengineering, University of Illinois at Chicago, 60607 Chicago, IL, USA. ${ }^{4}$ Department of Biomedical Engineering, Northwestern University, 60208 Evanston, IL, USA. ${ }^{5}$ Department of Mechanical Engineering, Northwestern University, 60208 Evanston, IL, USA. ${ }^{6}$ Sensory Motor Performance Program, Rehabilitation Institute of Chicago, 60611 Chicago, IL, USA. 'Department of Physiology, Northwestern University, 60611 Chicago, IL, USA.

\section{Received: 1 July 2014 Accepted: 25 September 2014}

\section{Published online: 31 October 2014}

\section{References}

1. Haugh AB, Pandyan AD, Johnson GR (2006) A systematic review of the Tardieu Scale for the measurement of spasticity. Disabil Rehabil 28(15):899-907, doi:10.1080/09638280500404305

2. Bohannon RW, Smith MB (1987) Interrater reliability of a modified Ashworth scale of muscle spasticity. Phys Ther 67(2):206-207

3. Jethwa A, Mink J, Macarthur C, Knights S, Fehlings T, Fehlings D (2010) Development of the Hypertonia Assessment Tool (HAT): a discriminative tool for hypertonia in children. Dev Med Child Neurol 52(5):e83-e87, doi:10.1111/.1.1469-8749.2009.03483.x

4. Brashear A, Zafonte R, Corcoran M, Galvez-Jimenez N, Gracies JM, Gordon MF, McAfee A, Ruffing K, Thompson B, Williams M, Lee CH, Turkel C (2002) Inter- and intrarater reliability of the Ashworth Scale and the Disability Assessment Scale in patients with upper-limb poststroke spasticity. Arch Phys Med Rehabil 83(10):1349-1354, doi:S0003999302000497

5. de Vlugt E, de Groot J, Schenkeveld K, Arendzen JH, van der Helm F, Meskers C (2010) The relation between neuromechanical parameters and Ashworth score in stroke patients. J NeuroEng Rehab 7(1):35

6. Mirbagheri MM, Alibiglou L, Thajchayapong M, Rymer WZ (2008) Muscle and reflex changes with varying joint angle in hemiparetic stroke. J NeuroEng Rehab 5:6-6

7. Piovesan D, Morasso P, Giannoni P, Casadio M (2012) Arm Stiffness during Assisted Movement after Stroke: the Influence of Visual Feedback and Training. In: IEEE Transactions on Neural Systems and Rehabilitation Engineering. doi:10.1109/TNSRE.2012.2226915

8. Li-Qun Z, Hyung-Soon P, Yupeng R (2009) Shoulder, elbow and wrist stiffness in passive movement and their independent control in voluntary movement post stroke. In: IEEE international conference on rehabilitation robotics (ICORR). Kyoto, pp 805-811, doi:10.1109/icorr.2009.5209489

9. Wood D, Burridge J, Wijck FV, McFadden C, Hitchcock R, Pandyan A, Haugh A, Salazar-Torres J, Swain I (2005) Biomechanical approaches applied to the lower and upper limb for the measurement of spasticity: a systematic review of the literature. Disabil Rehabil 27(1-2):19-33, doi:10.1080/09638280400014683

10. Dyer J-O, Maupas E, de Andrade MS, Bourbonnais D, Fleury J, Forget R (2009) Transmission in heteronymous spinal pathways is modified after stroke and related to motor incoordination. PLoS One 4(1):e4123, doi:10.1371/journal.pone.0004123

11. Piovesan D, Casadio M, Mussa-Ivaldi FA, Morasso P (2012) Comparing two computational mechanisms for explaining functional recovery in robottherapy of stroke survivors. In: 4th IEEE RAS \& EMBS international conference on biomedical robotics and biomechatronics (BioRob). Rome, pp 1488-1493, doi:10.1109/BioRob.2012.6290914

12. Mayer NH, Esquenazi A, Childers MK (1997) Common patterns of clinical motor dysfunction. Muscle Nerve Suppl 6:S21-S35

13. Biering-Sorensen F, Nielsen JB, Klinge K (2006) Spasticity-assessment: a review. Spinal Cord 44(12):708-722

14. Grow DI, Mengnan W, Locastro MJ, Arora SK, Bastian AJ, Okamura AM (2008) Haptic Simulation of Elbow Joint Spasticity. In: Symposium on Haptic Interfaces for Virtual Environment and Teleoperator Systems (Haptics 2008). Reno, pp 475-476, doi:10.1109/haptics.2008.4479997

15. Hyung-Soon P, Jonghyun K, Damiano DL (2012) Development of a Haptic Elbow Spasticity Simulator (HESS) for improving accuracy and reliability of clinical assessment of spasticity. IEEE Trans Neural Syst Rehabil Eng 20(3):361-370, doi:10.1109/tnsre.2012.2195330

16. Kim J-P, Ryu J (2010) Robustly stable haptic interaction control using an energybounding algorithm. Int J Rob Res 29(6):666-679, doi:10.1177/0278364909338770 
17. Carignan CR, Olsson PA (2004) Cooperative Control of Virtual Objects over the Internet using Force-Reflecting Master Arms. In: 2004 IEEE International Conference on Robotics and Automation, 2004. Proceedings. ICRA'04, Barcelona, vol 1222, pp 1221-1226. doi:10.1109/robot.2004.1307991

18. Mussa-Ivaldi FA (1992) From basis functions to basis fields: vector field approximation from sparse data. Biol Cybern 67(6):479-489

19. Mussa-Ivaldi FA, Giszter SF (1992) Vector field approximation: a computationa paradigm for motor control and learning. Biol Cybern 67(6):491-500

20. Melendez-Calderon A, Piovesan D, Mussa-Ivaldi FA (2013) Therapist discrimination of impaired muscle groups in simulated multi-joint hypertonia. In: 13th IEEE international conference on rehabilitation robotics (ICORR). Seattle, doi:10.1109/ICORR.2013.6650425

21. Piovesan D, Melendez-Calderon A, Mussa-Ivaldi FA (2013) Haptic Discrimination of Multijoint Dystonia and Spasticity in the Assessment of Hypertonia. In: 13th IEEE International Conference on Rehabilitation Robotics (ICORR). Seattle, doi:10.1109/ICORR.2013.6650449

22. Winter DA (2004) Biomechanics and motor control of human movement, 3rd edn. John Wiley \& Sons, New York

23. Hatze H (1977) A myocybernetic control model of skeletal muscle. Biol Cybernetics 25(2):103-119, doi:10.1007/bf00337268

24. Hatze H (1978) A general myocybernetic control model of skeletal muscle. Biol Cybernetics 28(3):143-157, doi:10.1007/bf00337136

25. Galiana L, Fung J, Kearney R (2005) Identification of intrinsic and reflex ankle stiffness components in stroke patients. Exp Brain Res 165(4):422-434

26. Granata KP, Wilson SE, Massimini AK, Gabriel R (2004) Active stiffness of the ankle in response to inertial and elastic loads. J Electromyogr Kinesiol 14(5):599-609, doi:10.1016/j.jelekin.2004.03.005 \$1050641104000458

27. Mirbagheri MM, Barbeau H, Kearney RE (2000) Intrinsic and reflex contributions to human ankle stiffness: variation with activation level and position. Exp Brain Res 135(4):423-436

28. Pisano F, Miscio G, Del Conte C, Pianca D, Candeloro E, Colombo R (2000) Quantitative measures of spasticity in post-stroke patients. Clin Neurophysiol 111(6):1015-1022, doi:S1388-2457(00)00289-3

29. Piovesan D, Casadio M, Mussa-Ivaldi FA, Morasso PG (2011) Multijoint arm stiffness during movements following stroke: implications for robot therapy. In: 2011 IEEE international conference on rehabilitation robotics (ICORR). Zurich, doi:10.1109/ICORR.2011.5975372

30. Kanade P, Scheidt RA (2009) Spatial mapping of posture-dependent endpoint forces in the hypertonic arm post-stroke. Doctoral dissertation, Marquette University, USA

31. Watts RL, Wiegner AW, Young RR (1986) Elastic properties of muscles measured at the elbow in man: II. Patients with parkinsonian rigidity. J Neurol Neurosurg Psychiatry 49(10):1177-1181

32. Given JD, Dewald JP, Rymer WZ (1995) Joint dependent passive stiffness in paretic and contralateral limbs of spastic patients with hemiparetic stroke. J Neurol Neurosurg Psychiatry 59(3):271-279, doi:10.1136/jnnp.59.3.271

33. Tan HZ, Durlach NI, Beauregard GL, Srinivasan MA (1995) Manual discrimination of compliance using active pinch grasp: the roles of force and work cues. Percept Psychophys 57(4):495-510

34. Youngung S, McMains S (2004) Evaluation of drawing on 3D surfaces with haptics. IEEE Comput Graph Appl 24(6):40-50, doi:10.1109/mcg.2004.49

35. Piovesan D, Melendez-Calderon A, Mussa-Ivaldi FA (2013) Haptic perception of multi-joint hypertonia during simulated patient-therapist physical tele-interaction. Conf Proc IEEE Eng Med Biol Soc 2013:4143-4147, doi:10.1109/EMBC.2013.6610457

36. Nicholson L, Adams R, Maher C (1997) Reliability of a discrimination measure for judgements of non-biological stiffness. Man Ther 2(3):150-156 doi:10.1054/math.1997.0295

37. Crutchfield CA (1976) The perception of tactile stimuli by physical therapy students before and after haptic training. Doctoral dissertation, West Virginia University, USA

38. Gibson JJ (1966) The senses considered as perceptual systems. Houghton Mifflin, Boston
39. Davidson PW, Whitson TT (1974) Haptic equivalence matching of curvature by blind and sighted humans. J Exp Psychol 102(4):687-690, doi:10.1037/h0036245

40. Soltero DE, Schwager M, Rus D (2012) Generating Informative Paths for Persistent Sensing in Unknown Environments. In: 2012 IEEE/RSJ International Conference on Intelligent Robots and Systems (IROS). Vilamoura, doi:10.1109/IROS.2012.6385730

doi:10.1186/s40638-014-0012-5

Cite this article as: Melendez-Calderon et al:: Enhanced assessment of limb neuro-mechanics via a haptic display. Robotics and Biomimetics 2014 1:12.

\section{Submit your manuscript to a SpringerOpen ${ }^{\odot}$ journal and benefit from:}

- Convenient online submission

- Rigorous peer review

- Immediate publication on acceptance

- Open access: articles freely available online

- High visibility within the field

- Retaining the copyright to your article

Submit your next manuscript at $>$ springeropen.com 